\title{
Pareto ratio and Pareto principle
}

\author{
Gennady A. Grachev \\ Southern Federal University, Rostov-on-Don, Russia \\ Email: grachev@sfedu.ru
}

\begin{abstract}
In the late 19th century, Vilfredo Pareto published the results of his observations of wealth inequality in Italy as a ratio in which the numerator is equal to the share of population ranked in descending order of wealth, and the denominator is the share of wealth of the population. Both the form of presentation of the concentration of inequality in the form of a visual ratio (the Pareto ratio), and an unexpectedly large inequality (80/20), which became known as the Pareto principle. In the 21 st century, a new concept was introduced - the generalized Pareto principle, which is understood as any proportion in which the sum of the numerator and denominator is $100 \%$. As a result, an extraordinary situation arose, when the empirical regularity began to be identified with the measure of its measurement. To resolve this confusion, the definition of Pareto ratio is provided in this work, based on a generally accepted concept of "wealthy" element of the system, as also an alternative explanation of the Pareto principle is proposed with the use of isoperimetric inequalities conceptually related with the principle of least action.
\end{abstract} Keywords: size inequality, Pareto curve, Pareto ratio, Pareto principle, asymmetry, isoperimetric inequality. 


\title{
Пропорция Парето и принцип Парето
}

\author{
Г.А. Грачёв \\ Южный Федеральный университет, Ростов-на-Дону, Россия \\ E-mail: grachev@sfedu.ru
}

\begin{abstract}
Аннотация
В конце XIX века Вильфредо Парето опубликовал результаты исследования неравенства богатства в Италии в виде отношения, в котором числитель равен доле населения, ранжированного по убыванию богатства, а знаменатель - доле приходящегося на него богатства. Новыми для того времени были как форма представления концентрации неравенства в виде наглядного отношения (пропорция Парето), так и неожиданно большое неравенство (20/80), которое стали называть принципом Парето. В XXI веке ввели в оборот новое понятие - обобщенный принцип Парето, под которым понимают любую пропорцию, у которой сумма числителя и знаменателя равна 100\%. В результате возникла неординарная ситуация, когда эмпирическую закономерность стали отождествлять с мерой ее измерения. В работе для устранения этой путаницы дано определение пропорции Парето исходя из общепризнанного понятия «богатый» элемент системы и предложен вариант объяснения принципа Парето с помощью изопериметрического неравенства, концептуально связанного с принципом наименьшего действия.
\end{abstract}

Ключевые слова: неравенство размеров, кривая Парето, пропорция Парето, принцип Парето, асимметрия, изопериметрическое неравенство. 


\section{1. Введение}

В конце XIX века Вильфредо Парето опубликовал результаты проведенного им исследования дифференциации богатства в Италии в виде отношения 20/80, в которой числитель равен доле населения, ранжированного по убыванию богатства, а знаменатель — доле приходящегося на него богатства [1]. Новыми для того времени были как форма представления концентрации неравенства в виде наглядного отношения (далее пропорция Парето), так и неожиданно большое неравенство. Дальнейшие исследования дифференциации размеров в системах показали, что пропорция 20/80 устойчиво проявляет себя не только в экономике, но и других областях знания. Многочисленные примеры этому можно найти в работах [2-4]. В середине XX века Джозеф Джуран, отдавая дань уважения Парето, назвал факт проявления пропорции 20/80 принципом Парето [5]. В начале XX века были созданы два важных инструмента для измерения неравенства доходов и богатства — кривая Лоренца [6] и коэффициент Джини [7]. Последний, несмотря на существенные недостатки [8], стал наиболее популярной мерой измерения концентрации неравенства. В дополнение к нему были разработаны другие показатели [9], включая коэффициент асимметрии и длину кривой Лоренца [10]. Пропорция Парето в этот период времени отошла на второй план. В то же время принцип Парето как эмпирическая закономерность всегда оставался в центре внимания [11-23]. Однако попытки объяснить его и, тем более доказать оказались тщетными. По мнению автора, основной причиной неудач стало отсутствие однозначного толкования понятия «пропорция Парето», без которого решить эту проблему невозможно. Вместо этого в XXI веке ввели в оборот новое понятие — «обобщенный принцип Парето», под которым понимают любую пропорцию, у которой сумма числителя и знаменателя равна $100 \%$ [24], что не корректно, так как принцип Парето по определению равен пропорции 20/80. Таким образом, возникла неординарная ситуация, когда эмпирическую закономерность — принцип Парето стали отождествлять с мерой измерения неравенства — пропорция Парето, что вносит путаницу при решении многих вопросов, в том числе определении нормы функционирования систем [25].

У данной работы две цели. Первая - определить понятие «пропорция Парето» при помощи ранее выработанного понятия «богатый» элемент системы [26]. Вторая объяснить принцип Парето с помощью изопериметрического неравенства, концептуально связанного с принципом наименьшего действия. 


\section{2. Пропорция Парето}

\section{1. Кривая Парето}

Пусть система состоит из $n$ элементов. Ранжируем элементы по убыванию размера и обозначим $w_{r}$ долю размера элемента с рангом $r$. Элементы такого рангового распределения удовлетворяют неравенству $w_{r} \leq w_{r-1}$. Доля накопленного размера в $k$ элементах системы с рангами $1,2, \ldots, k$, равна $S_{k}=\sum_{r=1}^{k} w_{r}$. График зависимости $S_{k}$ от доли элементов $\left(p_{k}=k / n\right)$ является дискретной функцией. При $n \rightarrow \infty \quad S_{k}=S\left(p_{k}\right)$ можно считать неотрицательной неубывающей дифференцируемой функцией $S(p)$, которую далее будем называть кривой Парето (РС). Из определения РС следует неравенство $p \leq S(p) \leq 1$ для $p \in[0,1]$ и граничные условия: $S(0)=0, S(1)=1$. РС однозначно связана с кривой Лоренца $(L)$ соотношением $S(p)=1-L(1-p)$.

Критическим показателем степени (или просто критическим показателем) РС, следуя [27], будем называть предел:

$$
\beta=\lim _{p \rightarrow 0} \frac{\ln S(p)}{\ln p} .
$$

Если предел существует, то в окрестности нуля $(p<<1)$ РС можно представить в виде $S(p) \sim C p^{\beta}, 0 \leq \beta \leq 1, C-$ константа. Данный степенной закон является точным в асимптотическом режиме $p \rightarrow 0$.

\section{2. Определение пропориии Парето}

Для определения понятия «пропорция Парето» используем следующее свойство РС. Средний по величине элемент системы находится в точке $\left(p_{\mu}\right)$, в которой производная $S^{\prime}\left(p_{\mu}\right)=1$ (Kakwani, 1980). Из этого свойства следует, что элементы системы можно разделить на два класса — класс «бедных», у которых $w\left(p_{r}\right)<w\left(p_{\mu}\right)$, и класс «богатых», удовлетворяющих неравенству $w\left(p_{r}\right) \geq w\left(p_{\mu}\right)$ [26]. Соответственно, доля «богатых» равна $p_{\mu}$, а доля приходящегося на них размера $-S\left(p_{\mu}\right)$. Пропорцию Парето определим как отношение $p_{\mu}$ к $S\left(p_{\mu}\right)$. Сумма числителя и знаменателя такой пропорции $A=p_{\mu}+S\left(p_{\mu}\right)$ может быть меньше, равна или больше единицы. Кривая Парето симметрична относительно диагонали ортогональной к эгалитарной линии, если $A=1$, имеет левостороннюю асимметрию, когда $A>1$ и правостороннюю асимметрию, когда $A<1[10]$. 
Таким образом, пропорция $p_{\mu} / S\left(p_{\mu}\right)$ не только однозначно делит элементы системы на «богатых» и «бедных», что делает представление о концентрации неравенства более понятным, но и позволяет по числителю и знаменателю пропорции определить асимметрию РС.

\section{3. Принцип Парето}

\section{3. Модель симметричных PC}

Учитывая, что концептуально изопериметрическое неравенство связано с принципом наименьшего действия, объясняющим, почему масляные пятна на поверхности воды имеют форму окружности, а капля воды - форму шара, можно предположить, что у систем, функционирующих без ограничений, кривая Парето должна быть симметричной относительно ортогональной диагонали. Подтверждением этому служит закон Гибрата [28] и многочисленные эмпирические исследования (см., например, [29], [11], [30]).

Наиболее простой для вычислений и в то же время адекватно описывающей симметричные РС систем разной природы [4,30-37] является однопараметрическая модель РС распределения Burr III [38]:

$$
S(p ; \alpha)=\left(1-(1-p)^{\alpha}\right)^{\frac{1}{\alpha}}, 1 \leq \alpha .
$$

Подставляя (2) в (1), находим: $\beta=1 / \alpha$, что говорит о том, что параметр $\alpha$ определяет не только форму РС, но и может трактоваться, как фрактальная размерность системы.

Дифференцируя (2), получаем:

$$
\frac{d S(p ; \alpha)}{d p}=\left[\frac{1-p}{S(p ; \alpha)}\right]^{\alpha-1} .
$$

Приравняв (3) единице, после очевидных преобразований находим:

$$
p_{\mu}=1-0.5^{\frac{1}{\alpha}}
$$

Из (4) получаем:

$$
\alpha=\frac{\ln 0.5}{\ln \left(1-p_{\mu}\right)}
$$

Подставляя в (5) $p_{\mu}=0.2$, находим $\alpha \approx 3.1$. Подставляя в (4) $\alpha=3$, получаем: $p_{\mu}=0.206, S_{\mu}=0.794$. Таким образом, системам с тремя степенями свободы $(\alpha=3)$ соответствует приближенная пропорция 21/79, что расходится с пропорцией 20/80. Однако, учитывая погрешность статистических данных, с которыми имел дело Парето, 
можно предположить, что полученная им пропорция 20/80 является результатом округления чисел пропорции 20.6/79.4.

Вычисляя коэффициент Джини, находим:

$$
G=2 \int_{0}^{1} S(p) d p-1=\frac{\Gamma(1 / \alpha)^{2}}{\alpha \Gamma(2 / \alpha)}-1, \alpha \geq 1 .
$$

Зависимость $p_{\mu}$ от коэффициента Джини показана на Fig. 1.

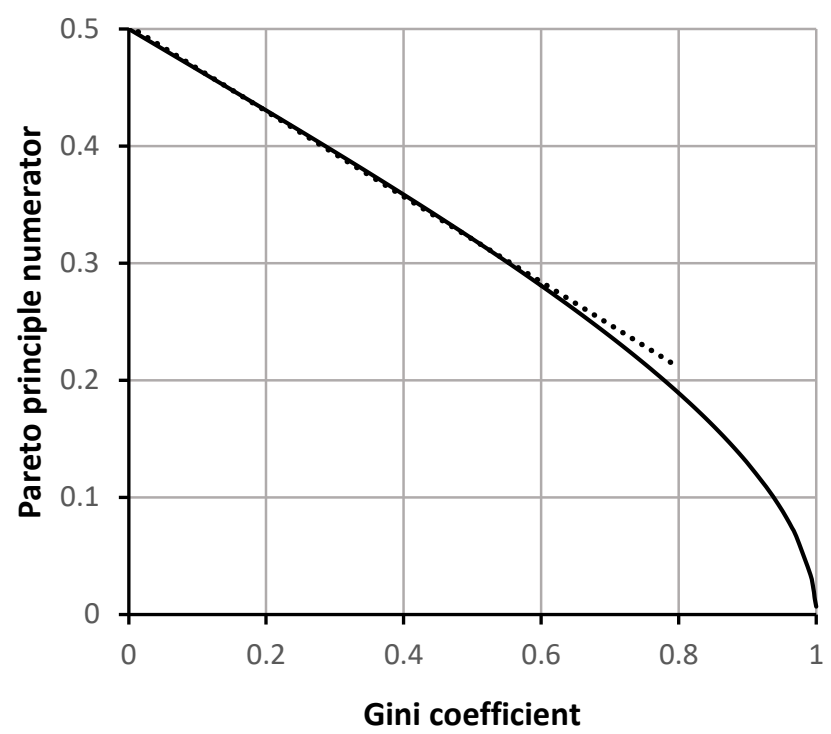

Fig. 1. Зависимость числителя пропорции Парето от коэффициента Джини

Аппроксимируя эту зависимость полиномом первой степени (пунктир), получаем:

$$
p_{\mu}=0.503-0.364 G, \quad G \leq 0.6 \text {. }
$$

Существенным здесь является то, что линейная зависимость $p_{\mu}$ от $G$ для значений $G \leq 0.6$ совпадает с эмпирической зависимостью, полученной в работах $[39,40]$, что еще раз подтверждает соответствие модели (6) системам разной природы.

\section{4. Объяснение принципа Парето}

Изопериметрическое неравенство — неравенство между объемом $V$ области в евклидовом пространстве $E^{n}, n \geq 2$, и $(n-1)$-мерной площадью $P$ гиперповерхности, ограничивающей эту область, удовлетворяет неравенству:

$$
Q_{n}=\frac{n^{n} v_{n} V^{n-1}}{P^{n}} \leq 1
$$

где $v_{n}$ - объем единичного $n$-мерного шара, $Q_{n}$ - изопериметрический коэффициент.

Для плоской фигуры, ограниченной кривой Парето и эгалитарной линией $(\mathrm{n}=2)$, изопериметрическое неравенство имеет вид: 


$$
Q_{2}=\frac{2 \pi G}{P^{2}} \leq 1
$$

где $P=l+\sqrt{2}$ - периметр плоской фигуры, $l=\int_{0}^{1} \sqrt{1+\left(S^{\prime}\right)^{2}} d p-$ длина РС.

Графики зависимости $Q_{2}$ и коэффициента Джини от длины нормированного периметра показаны на Fig. 2.

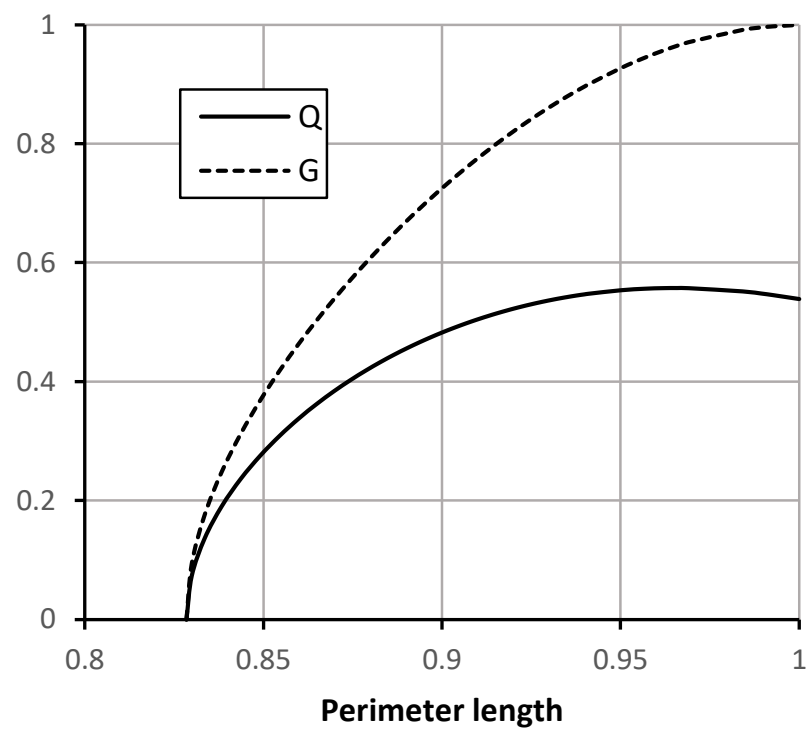

Fig. 2. Зависимости и G от длины нормированного периметра

Из представленных на Fig. 2 зависимостей видно, что $Q_{2}(P)$ имеет максимум в точке $(0.96,0.56)$, которой соответствуют $G \approx 0.96, p_{\mu}=0.068$ (см. Fig. 1) и $\alpha=9.8$. Таким образом, неравенство размеров в системах может развиваться до пропорции 7/93. Однако у природных систем, имеющих размерности 1, 2 и 3, максимум неравенства равен $21 / 79$.

\section{4. Выводы}

Подводя итоги, можно констатировать, что использование среднего для деления элементов системы на «богатые» и «бедные» устраняет неоднозначность понятия «пропорция Парето». Установлено, что принцип Парето проявляет себя только в системах с тремя степенями свободы и симметричными кривыми Парето. Неравенство суммы числителя и знаменателя пропорции Парето 100\% говорит об асимметрии РС. Из изопериметрического неравенства следует, что эволюционное развитие систем должно сопровождаться уменьшением асимметрии РС и ростом неравенства размеров до пропорции 20/80, которая является верхней границей неравенства в физических системах. 


\section{Литература}

[1] V. Pareto, Cours d'Économie Politique, Vol. I. Pp. 430. I896. Vol. II. Pp. 426. I897. Lausanne: F. Rouge, Ann. Am. Acad. Pol. Soc. Sci. 9 (1897) 128-131. https://doi.org/10.1177/000271629700900314.

[2] G.K. Zipf, Human behavior and the principle of least effort, Cambridge, (Mass.): Addison-Wesley, 1949. https://doi.org/10.1002/1097-4679(195007)6:3<306::aidjclp2270060331>3.0.co;2-7.

[3] R. Koch, The 80/20 Principle: The Secret of Achieving More with Less, Nicholas Brealey Publishing, London, 1997.

[4] Г.А. Грачёв, Моделирование принципа Парето, Южный федеральный ун-т, Ростов-на-Дону, 2011.

[5] J.M. Juran, The Non-Pareto Principle; mea culpa, Qual. Prog. 8 (1975) 8-9.

[6] M.O. Lorenz, Methods of Measuring the Concentration of Wealth, 1905.

[7] C. Gini, Measurement of Inequality of Incomes, Econ. J. 31 (1921) 124. https://doi.org/10.2307/2223319.

[8] L. Osberg, On the Limitations of Some Current Usages of the Gini Index, Rev. Income Wealth. 63 (2017) 574-584. https://doi.org/10.1111/roiw.12256.

[9] I.I. Eliazar, I.M. Sokolov, Measuring statistical evenness: A panoramic overview, Phys. A Stat. Mech. Its Appl. 391 (2012) 1323-1353.

[10] N. Kakwani, Income inequality and poverty: methods of estimation and policy applications, Published for the World Bank [by] Oxford University Press, 1980.

[11] D. Herron, Industrial engineering applications of abc curves, AIIE Trans. 8 (1976) 210 218. https://doi.org/10.1080/05695557608975069.

[12] Q.L. Burrell, The 80/20 rule: Library lore or statistical law?, J. Doc. 41 (1985) 24. https://doi.org/10.1108/eb026772.

[13] H. Kumakura, Market Structure Analysis Using Birth and Asymmetric Growth of Products Based on a Mechanism of the 80/20 Law: Why and How the 80/20 Law Emerges, 2010.

[14] M. Goeminne, T. Mens, Evidence for the Pareto principle in Open Source Software Activit, in: First Int. Work. Model. Softw. Migr. (MDSM 2011) Fifth Int. Work. Softw. Qual. Maintainab. (SQM 2011), 2011: pp. 74-82.

[15] K. Tanabe, Pareto's 80/20 rule and the Gaussian distribution, Phys. A Stat. Mech. Its Appl. 510 (2018) 635-640. https://doi.org/10.1016/j.physa.2018.07.023.

[16] L. Egghe, On the 80/20 rule, Scientometrics. 10 (1986) 55-68. 
https://doi.org/10.1007/BF02016860.

[17] Y.S. Chen, P.P. Chong, M.Y. Tong, Mathematical and computer modelling of the Pareto principle, Math. Comput. Model. (1994). https://doi.org/10.1016/0895-7177(94)900418.

[18] J.C.H. Chen, P.P. Chong, Y.S. Chen, Decision criteria consolidation: A theoretical foundation of pareto principle to porter's competitive forces, J. Organ. Comput. Electron. Commer. 11 (2001) 1-14. https://doi.org/10.1207/S15327744JOCE1101_01.

[19] A. Ultsch, Proof of Pareto's 80/20 Law and Precise Limits for ABC-Analysis, 2002.

[20] M.E.J. Newman, Power laws, Pareto distributions and Zipf's law, Contemp. Phys. 46 (2005) 323-351. https://doi.org/10.1016/j.cities.2012.03.001.

[21] И.Г. Царев, О моделировании распределения доходов в обществе, Прикладная Статистика. 3 (2008) 43-51.

[22] S. Lipovetsky, Pareto 80/20 law: Derivation via random partitioning, Int. J. Math. Educ. Sci. Technol. 40 (2009) 271-277. https://doi.org/10.1080/00207390802213609.

[23] M. Hardy, Pareto's Law, Math. Intell. 32 (2010) 38-43. https://doi.org/10.1007/s00283010-9159-2.

[24] S. Banerjee, B.K. Chakrabarti, M. Mitra, S. Mutuswami, On the Kolkata index as a measure of income inequality, Phys. A Stat. Mech. Its Appl. 545 (C) (2020). https://doi.org/10.1016/j.physa.2019.123178.

[25] Y.L. Klimontovich, Relative ordering criteria in open systems, Physics-Uspekhi. 39 (1996) 1169-1179. https://doi.org/10.1070/PU1996v039n11ABEH000181.

[26] D. Acemoglu, D. Autor, Skills, tasks and technologies: implications for employment and $\begin{array}{llll}\text { earnings, } & \text { Cambridge, } & \text { MA } & \text { 02138, }\end{array}$ https://doi.org/http://www.nber.org/papers/w16082.

[27] H. Stanley, Introduction to phase transitions and critical phenomena, Oxford University Press, New York, 1971.

[28] R. Gibrat, Les Inégalités Économiques: Applications, aux Inégalités des Richesses, a la Concentration des Entreprises, aux Populations des Villes, aux Statistiques des Familles, etc.: d'une Loi Nouvelle la Loi de l'Effet Proportionnel, Recueil Sirey, Paris, 1931.

[29] P.E. Hart, S.J. Prais, The Analysis of Business Concentration: A Statistical Approach, J. R. Stat. Soc. Ser. A. 119 (1956) 150. https://doi.org/10.2307/2342882.

[30] G.A. Grachev, Size Distribution of States, Counties, and Cities in the USA: New Inequality Form Information, Preprints.Ru. (2020). https://doi.org/https://doi.org/10.24108/preprints-3112131.

[31] Г.А. Грачёв, Системные закономерности неравенства доходов населения, 
Экономические Науки. 8(57) (2009) 327-331.

[32] Г.А. Грачёв, Модель оптимального состояния системы городского расселения, Известия Российской Академии Наук. Серия Географическая. 3 (2010) 46-51.

[33] Г.А. Грачёв, К оценке политической стабильности по результатам голосования на выборах, Полис. Политические Исследования. 5 (2011) 123-127.

[34] Г.А. Грачёв, К прогнозированию оптимальной структуры российской банковской системы, Проблемы Прогнозирования. 22 (2011) 103-109.

[35] Г.А. Грачёв, К анализу структурной эффективности государственных союзов, Экономические Науки. 6(79) (2011) 212-215.

[36] Г.А. Грачёв, Оценка политической стабильности на прошедших выборах парламента и президента России, Полис. Политические Исследования. 3 (2012) $30-35$.

[37] Г.А. Грачёв, Управление структурой денежных доходов населения на основе принципа Парето, Terra Econ. 11 (2013) 57-63.

[38] I.W. Burr, Cumulative Frequency Functions, Ann. Math. Stat. 13 (1942) 215-232. https://doi.org/10.1214/aoms/1177731607.

[39] A. Ghosh, N. Chattopadhyay, B.K. Chakrabarti, Inequality in societies, academic institutions and science journals: Gini and k-indices, Phys. A Stat. Mech. Its Appl. 410 (2014) 30-34. https://doi.org/10.1016/j.physa.2014.05.026.

[40] A. Chatterjee, A. Ghosh, B.K. Chakrabarti, Socio-economic inequality: Relationship between Gini and Kolkata indices, Phys. A Stat. Mech. Its Appl. 465 (2017) 583-595. https://doi.org/10.1016/j.physa.2016.09.027. 